\title{
Reconstruction of low speed rear-end collisions - Technical means of assessing cervical spine injuries
}

\author{
Dipl.-Ing. (FH) M artin Hitzemann*
}

I recent years an increasing number of cervical spine injuries to vehicle occupants in collisions at comparatively low speeds have been reported to insurance companies and in civil litigation. The reported injuries occur in both rear-end and side impact collisions. However, the following presentation will deal primarily with rear-end collisions. The later, in detail assessment of the issues explained here can only be performed through interdisciplinary teamwork involving technical and biomechanical/medical experts.

The technical expert is particularly confronted by the following questions:

- A t what speed was the bullet (striking) vehicle travelling?

- What change of velocity did the target vehicle experience?

- What acceleration/deceleration was exerted on the vehicle and its occupants?

The biomechanical/medical expert then has to clarify the extent to which the forces of acceleration and changes of vel ocity of the target vehicle are causal factors for the reported injuries and their duration. A ssessment of the velocity curve of the target vehicle and the resultant deformational loads on the vehicle depend upon the vehicle related information made available. The precise extent of the damage to both the target vehicle and the bullet vehicle are crucial criteria.

The collision can be assessed by means of the law of the conservation of momentum. This means that not only the masses of the vehicles but also their structural stiffness values have to be estimated in order to define the severity of the impact which is included in the consideration of momentum.

However, this pure consideration of momentum can be applied only if the collision involves low speeds which also result in correspondingly slight, predominantly elastic, deformation of the vehicles.

The assessment of the deformation energy is difficult due to the fact that large elastic deformation energies occur in modern bumper designs. In cases where only slight vehicle deformation has occurred, an equivalent barrier impact speed (EES - energy equivalent speed), which has to be considered in the reconstruction, cannot be included with sufficient accuracy. The EES estimation method is not suitable in this case but the so-called K-value (impact severity) becomes very important.
Experiments conducted by the M unich branch of DEKRA A utomobil AG in conjunction with Munich University of A pplied Sciences (Fachhochschule M ünchen) showed that for bumper designs consisting of sheet metal parts, the K-values lay in a range from approximately 0.14 to 0.32 , and in vehicle groupings where at least one bumper was equipped with elastic impact-absorbing elements, the K-values were determined within a range from 0.23 to 0.54 . The $\mathrm{K}$-values, and hence the figure for the impact severity of the vehicles involved in the crash, decrease as the plastic deformation of the vehicles increases. Consequently, in collisions involving only minor deformation of the vehicles in the predominantly elastic range, the $\mathrm{K}$-values are located in the upper limit of the range mentioned above. In contrast, in collisions where the deformations are clearly of a plastic nature, the deformation work has to be considered in the form of EES values. In these cases, considerations of purely momentum are not physically correct, as one part of the energy introduced by the collision would remain ignored. In this respect, the reconstruction method should be chosen depending on the available information and the documented damage.

In terms of the acceleration/deceleration acting on the vehicle, it is also important to know to what extent braking was applied to the target vehicle. A vehicle additionally retarded by the service or hand brake will decelerate the bullet vehicle more than in the case of a free moving target vehicle. The result of this is that the deformation to the rear end will be greater for a vehicle with the brakes applied than for one without. However, the change in velocity and acceleration acting through the collision are lower than for an unbraked vehicle.

The technical reconstruction can only determine the velocity changes/vehicle acceleration of the vehicle. Experiments have shown that the acceleration/deceleration values of the occupants, which were measured at chest and head level by sensors, occur at a later stage than the vehicle acceleration/deceleration.

A t low collision speeds, the values of occupant acceleration/deceleration, particularly at head level, are of a similar magnitude to those of the vehicle. In collisions with significant degrees of deformation, the disproportionately high head acceleration is determined due to the lever effect between the surface of the occupant's seat and the head. H owever, the max-

*DEKRA A utomobil G mbH, Vandalenweg 10, 20097, H amburg, G ermany 
imum value cannot be used for the assessment of the loading of the cervical spine because these maximum values were measured only when the head touched the head restraint. The crucial factor for the loading is the mean head acceleration over the impact period.

A different acceleration/deceleration history is present for the vehicle and its occupants if the impact occurs at an angle. In this case, significant glance-off effects can occur on the colliding vehicles. A $s$ a result of this, low changes of velocity are found, particularly in side-impact collisions, although one result of collision analysis, namely that of impact momentum occurring at a defined angle on the vehicle, can in fact be used for determining the occupant loading. The impact momentum acting on the vehicle can be transferred to the vehicle occupants so that corresponding loading values can be determined in this case as well.

From the technical view point, therefore, it is possible to use photographs and reports of damage in order to reconstruct acceleration/deceleration values acting on vehicles, which can then be employed for the biomechanical/medical assessment.

This material was presented at the International C ongress on W hiplash A ssociated Disorders, Berne, Switzerland, M arch 8 to 10, 2001 . The paper appeared originally in the book "W hiplash A ssociated D isorders" - medical, biomechanical and legal aspects, published by Staempfli Publishers Ltd, Berne 2002. The paper is published in N orth A merica in Pain Research \& M anagement with the permission of Staempfli Publishers Ltd. 


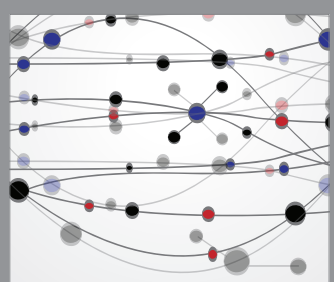

The Scientific World Journal
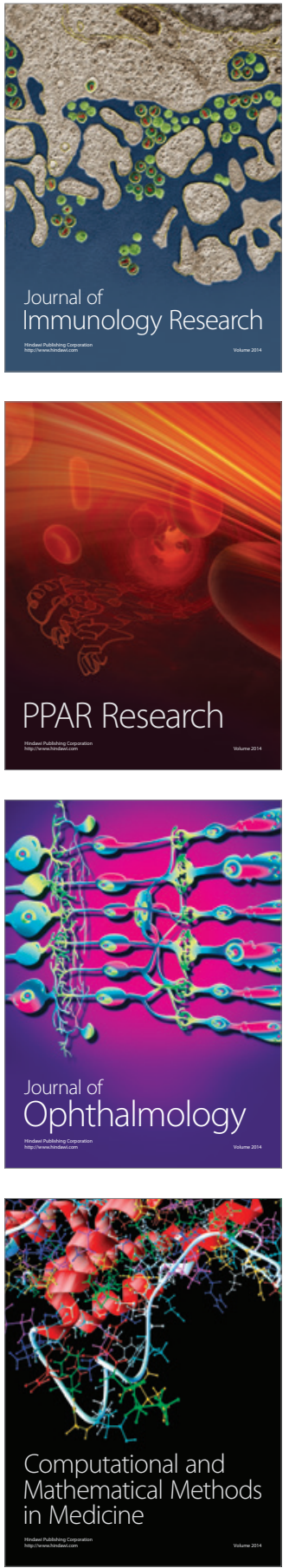

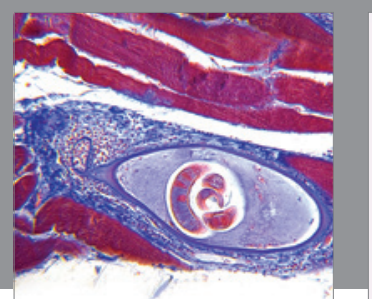

Gastroenterology Research and Practice

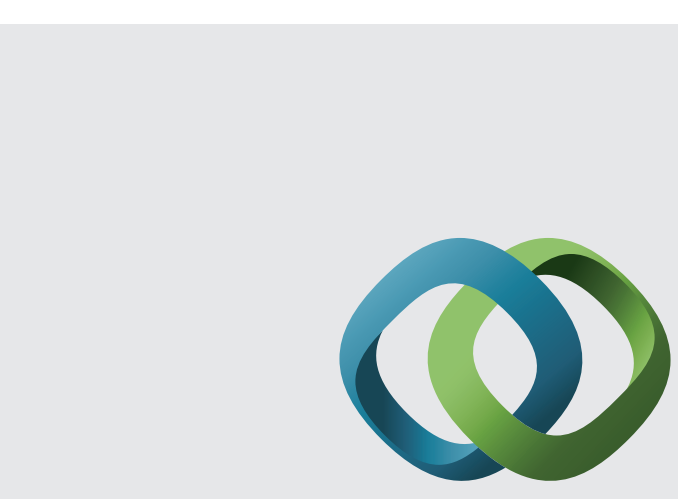

\section{Hindawi}

Submit your manuscripts at

http://www.hindawi.com
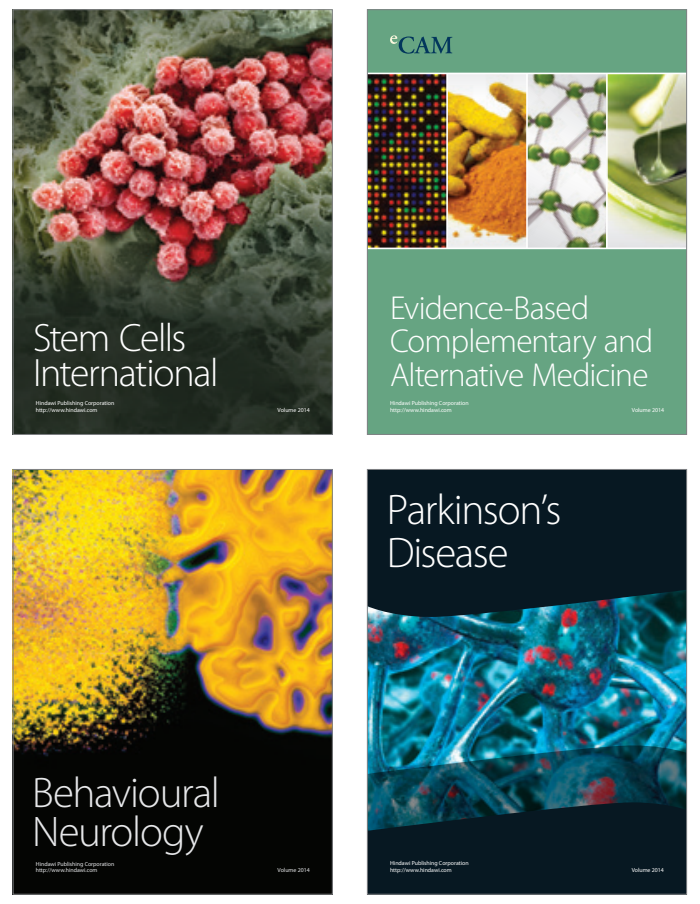


Disease Markers
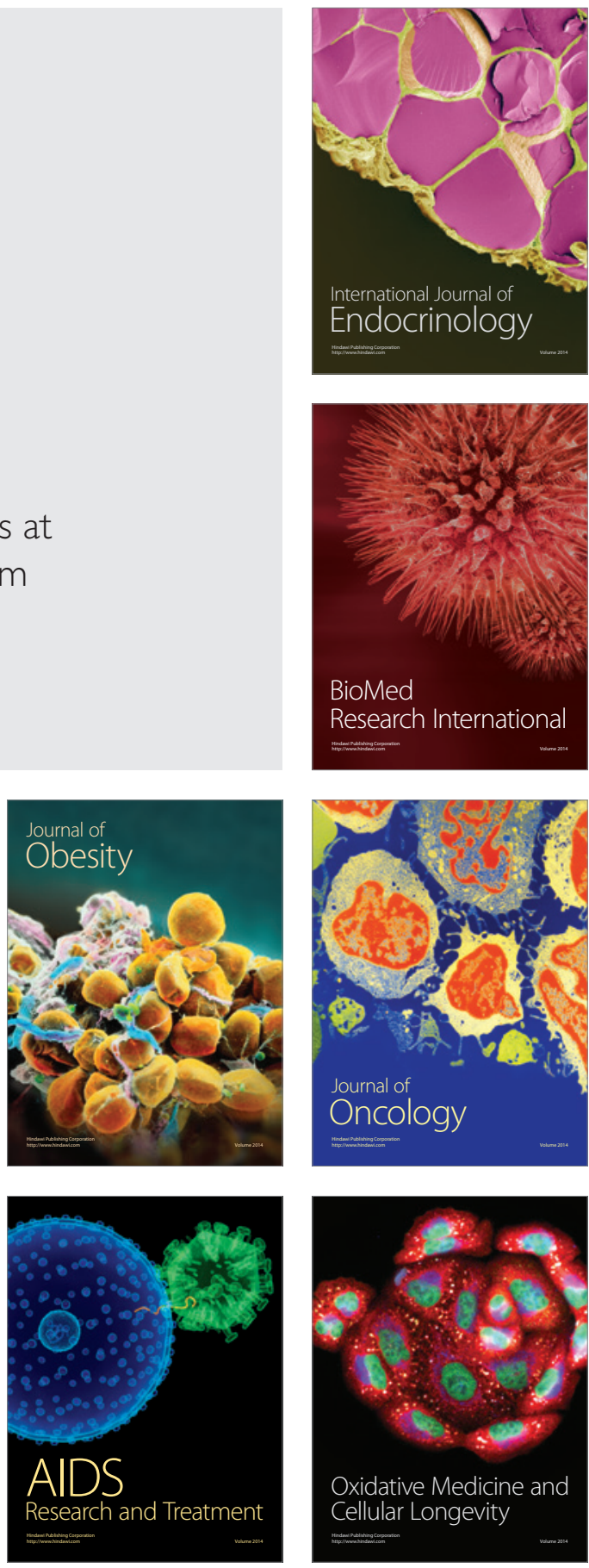